\title{
Educational Needs of Patients with Inflammatory Bowel Disease (IBD) and Non- Adherence to Medical Therapy-A Qualitative Study
}

\author{
Wheat $\mathrm{CL}^{*}$, Megan Maass, Beth Devine, Timothy Thornton, David Grembowski and Cynthia W Ko
}

Department of Health Services, University of Washington, Seattle, USA.

*Corresponding author: Chelle L Wheat, Department of Health Services, University of Washington, Seattle, USA, Tel: 2063105984; E-mail: wheatc@uw.edu

Received date: March 02, 2016; Accepted date: April 13, 2016; Published date: April 20, 2016

Copyright: (C) 2016 Wheat CL, et al. This is an open-access article distributed under the terms of the Creative Commons Attribution License, which permits unrestricted use, distribution, and reproduction in any medium, provided the original author and source are credited.

\begin{abstract}
Objective: Patients with IBD are at risk for serious complications when their disease is poorly managed. Patient non-adherence to medical therapy contributes to suboptimal outcomes, but may be modified through improved education. The primary aim of this study is to identify educational needs, and barriers and factors associated with non-adherence among inflammatory bowel disease (IBD) patients.
\end{abstract}

Methods: Eighteen IBD patients and ten IBD providers were recruited. Semi-structured interviews were conducted and a qualitative framework approach used to identify patient educational needs, barriers to obtaining information, and factors associated with non-adherence with medical therapy.

Results: Prevention of IBD symptoms and factors contributing to development of IBD were the most frequently identified patient educational needs. Both providers and patients identified diet and nutrition, as well as access to general information about IBD, as important areas of education. Common barriers to obtaining or conveying information for patients and providers included: information oversaturation, ineffective provider communication skills, and lack of provider time. Factors that impact patient comprehension and decision making were also identified. Providers frequently believed that patient non-adherence is associated with lack of current symptoms or denial of their chronic condition.

Conclusion: Our findings highlight several deficits in knowledge in IBD patients. We also identify factors associated with IBD patient comprehension, decision making, and non-adherence to therapy. These results can be used to develop targeted educational resources to improve adherence among IBD patients. We propose that patient self-management programs are potentially effective educational interventions that warrant further study in IBD.

Keywords: Inflammatory bowel disease; Crohn's disease; Ulcerative colitis; Qualitative study; Educational needs; Non-adherence; Educational resources

\section{Introduction}

Inflammatory bowel disease (IBD) is a group of autoimmune disorders of the gastrointestinal tract, most commonly ulcerative colitis (UC) and Crohn's disease (CD). IBD can be debilitating to those afflicted, and adverse effects from medical and/or surgical therapy may further contribute to a reduction in quality of life. Poorly controlled disease increases the risk for serious and often irreversible negative outcomes including death [1-5]. Patient non-adherence to medical therapy may contribute to suboptimal disease control and complications. Non-adherence to medical therapy may be associated with demographic factors such as gender (male) and marital status (single) [6]. Individuals may forego medical and/or surgical therapy due to inconvenience, costs, lack of understanding, or fear of side effects from therapy. Inconvenience and cost of therapy are difficult to address due to their limited mutability, but fear of adverse effects from therapy and lack of understanding of available treatment options are factors that may be amenable to change. Education specific to IBD, its effects, and its management, can potentially increase patient adherence and improve outcomes for these patients [7]. Current evidence suggests that many individuals with IBD lack the desired information about their disease [8-11]. Specific areas of interest to IBD patients include: prevention and management of IBD symptoms, complications related to IBD, long term prognosis, risk of cancer and mortality, alternative therapies, risks from pharmacotherapy, potential new therapies, and fertility [8-13]. These information needs were identified through surveys administered to specific patient populations (e.g. newly diagnosed); however, to our knowledge an intensive qualitative study in a diverse IBD patient population specifically exploring this topic has not been published. The primary aim of this study is to determine the perceived educational needs of IBD patients (i.e. the information about issues that a patient desires to understand such as their disease process, prognosis, and therapeutic options), and to identify areas where providers believe patient understanding is critical. This information can be used to develop novel educational resources to facilitate patient adherence to therapy. A further aim is to describe the barriers for patients and providers in obtaining or conveying desired information. Lastly, we aim to identify barriers and facilitators to patient comprehension, decision making, and adherence to therapy recommendations.

\section{Methods}

We identified and interviewed ten providers (MD, ARNP, RN) who care for patients with IBD at the University of Washington Medical Center (UWMC), Harborview Medical Center (HMC, a safety net 
Citation: Wheat CL, Maass M, Devine B, Thornton TY, Grembowski D, et al. (2016) Educational Needs of Patients with Inflammatory Bowel Disease (IBD) and Non-Adherence to Medical Therapy-A Qualitative Study. J Inflam Bowel Dis \& Disord 1: 106.

Page 2 of 8

hospital) and Veteran's Administration Puget Sound Health Care System (VA) in 2015. HMC is a public hospital that serves many patients with lower education and income levels compared to other healthcare facilities in the area (including UWMC). Providers were identified through publicly available online directories and contacted via email. In addition, eighteen patients with IBD who receive care at UWMC or HMC were interviewed. Patients were identified through their providers and asked for their verbal consent to be contacted by the lead researcher $(\mathrm{CW})$. All interviewees provided oral assent prior to being interviewed. Patient participants were oversampled from HMC due to known differences in education level, income level, and other patient characteristics (e.g. consistency of care, patient nonadherence to therapy) between the institutions. Two interview guides were utilized for this study, one for the patient population (Supplemental Appendix 1) and one for the providers (Supplemental Appendix 2). We developed our patient interview guide with feedback from a patient with a non-IBD related autoimmune condition, a gastroenterologist, and a patient advocate. Interviews lasted approximately thirty minutes, and were conducted by phone or inperson with the lead researcher. All interviews were recorded with permission and transcribed. The lead researcher did not have a prior relationship with any of the patient interviewees. Patient interviews were conducted until reaching information saturation $[14,15]$. Provider interviews were conducted until reaching our predetermined target of ten interviews. Although no existing formal analytical method for sample size determination in qualitative research is available, published literature suggests that twelve interviews provides approximately $92 \%$ of the information desired, and when utilizing knowledgeable individuals (such as IBD providers) ten or less participants is often sufficient $[14,15]$. Responses to the interview questions formed the basis for the qualitative analysis. We utilized a framework approach to guide our data analysis methodology $[15,16]$. Each interview was independently coded by two researchers (CW and $\mathrm{MM}$ ), the themes identified, and the data reviewed until consensus. Data were collected, stored, and analyzed using ATLAS.ti (ATLAS.ti Scientific Software Development GmbH Version 7.0). Themes were captured once per interview question; however repeating themes were categorized separately for the same respondent if readdressed in another question in order to detail the density of certain themes. Means with standard deviations were calculated for continuous participant characteristics, and proportions for categorical measures. Statistical analyses of descriptive characteristics were conducted using Stata MP 13.0 (Stata Corp. College Station, TX). The study was determined to be exempt from review by the University of Washington's Human Subjects Division. All respondents were assured of confidentiality, and informed that participation was voluntary. Interviewees were compensated a nominal amount ( $\$ 10.00$ Starbucks $\odot$ gift card) for their involvement.

\section{Results}

$77.8 \%$ of patient participants received their IBD care at HMC, and the majority were male (61.1\%) (Table 1$)$. Their mean age was $31.6+/$ 9.8 years and their average disease duration was $8.6+/-7.2$ years. Among the provider participants, $50 \%$ primarily practiced at UWMC and there was equal representation of men and women. Their average length of experience treating patients with IBD was $5.7+/-2.3$ years.

\begin{tabular}{|c|c|c|c|}
\hline & Patients ( $\mathrm{N}=18$ ) & & Providers $(\mathrm{N}=10)$ \\
\hline Institution-no. (\%) & & Institution-no. (\%) & \\
\hline UWMC & $4(22.2)$ & UWMC & $5(50.0)$ \\
\hline HMC & $14(77.8)$ & HMC & $3(30.0)$ \\
\hline VA & $0(0.0)$ & $V A^{*}$ & $2(20.0)$ \\
\hline Interview mechanism-no. (\%) & & Interview mechanism-no. (\%) & \\
\hline In-person & $8(44.4)$ & In-person & $10(100.0)$ \\
\hline Phone & $10(55.6)$ & Phone & $0(0.0)$ \\
\hline Sex-no. (\%) & & Sex-no. (\%) & \\
\hline Men & $11(61.1)$ & Men & $5(50.0)$ \\
\hline Women & $7(38.9)$ & Women & $5(50.0)$ \\
\hline Education level-no. (\%) & & Role-no. (\%) & \\
\hline High School & $3(16.7)$ & Fellow (MD) & $2(20.0)$ \\
\hline Technical School & $1(5.5)$ & Attending (MD) & $4(40.0)$ \\
\hline College & $14(77.8)$ & Nurse Practioner (NP) & $1(10.0)$ \\
\hline Age (years)-mean (SD) & $31.6(9.8)$ & Registered Nurse (RN) & $3(30.0)$ \\
\hline Disease duration (years)-mean (SD) & $8.6(7.2)$ & Length of IBD experience (years)-mean (SD) & $5.7(2.3)$ \\
\hline Past surgical history-no. (\%) & $7(38.9)$ & & \\
\hline
\end{tabular}


Citation: Wheat CL, Maass M, Devine B, Thornton TY, Grembowski D, et al. (2016) Educational Needs of Patients with Inflammatory Bowel Disease (IBD) and Non-Adherence to Medical Therapy-A Qualitative Study. J Inflam Bowel Dis \& Disord 1: 106.

Page 3 of 8

\begin{tabular}{|l|l|l|l|}
\hline Current biologic therapy -no. (\%) & $15(83.3)$ & & \\
\hline Current immunomodulator therapy-no. (\%)** & $14(77.8)$ & & \\
\hline Current steroid therapy-no. (\%) & $3(16.7)$ & & \\
\hline Current mesalamine/sulfasalazine therapy-no. (\%) & $3(16.7)$ & & \\
\hline Past biologic therapy-no. (\%) & $6(33.3)$ & & \\
\hline Past immunomodulator *therapy-no. (\%) & $3(16.7)$ & & \\
\hline Past steroid therapy-no. (\%) & $11(61.1)$ & & \\
\hline Past mesalamine/sulfasalazine therapy-no. (\%) & $5(27.8)$ & & \\
\hline
\end{tabular}

Table 1: Patient and provider demographic characteristics.

\section{Educational needs}

The most frequently conveyed educational need by IBD patients was prevention of IBD-related symptoms $(\mathrm{n}=12)$ (Table 2).

"Being able to piece together what's going to make my life as livable as it can be and my disease as manageable as it will be."

In addition, information about 0074 he prevention or cause of IBD was recurrently expressed by respondents $(n=11)$.

"It would be really interesting to me to find out what's the mechanism that causes the inflammatory process in the first place."

Expected medication effects and/or information related to medication management $(n=9)$, as well as natural remedies for IBD $(\mathrm{n}=9)$, were both common themes discussed.

"Making sure that there are some better options out there in case the meds that I am using are not working." (A patient discussing the importance of information regarding medication management).

"I feel like there is more that can be done than just putting someone on medication." (A patient expressing their desire for natural therapeutic options to treat their IBD).
Diet and nutritional concerns were especially important to many patients, regardless of whether they had newly diagnosed or established IBD $(\mathrm{n}=8)$.

"I wish I knew about diet more than anything else."

Other respondents thought that information about IBD in general, including its natural progression and the effects of the inflammatory process, would be valuable $(n=5)$. In addition, some patients were especially concerned about their own prognosis $(n=4)$. Lastly, some participants were interested in information about new therapies and technological advancements related to $\operatorname{IBD}(\mathrm{n}=2)$, and how to access resources to obtain their medical care and/or IBD medication $(\mathrm{n}=1)$.

"it would be interesting to hear more about new techniques of diagnosis, and new treatment options from my doctors."

Table 2 also details critical concepts for IBD patients to understand as identified by providers. The importance of adherence to medication $(n=13)$ and an understanding of IBD in general $(n=13)$ were the two most commonly expressed areas that providers feel are crucial for IBD patients to understand.

\begin{tabular}{|l|l|}
\hline Patient Identified Educational Needs & Frequency of occurrence \\
\hline Educational Need & 12 \\
\hline The prevention of symptoms related to IBD & 11 \\
\hline The prevention of IBD or information related to the cause of IBD & 9 \\
\hline Expected medication effects and/or information related to medication management & 9 \\
\hline Natural remedies for IBD & 8 \\
\hline Diet and nutrition & 9 \\
\hline IBD in general & 5 \\
\hline Disease prognosis & 4 \\
\hline New therapies and technological advancements related to IBD & 2 \\
\hline Accessing resources to obtain medical care and/or IBD pharmacotherapy & 1 \\
\hline Provider Identified Critical Understanding Areas for Patients & \\
\hline
\end{tabular}


Citation: Wheat CL, Maass M, Devine B, Thornton TY, Grembowski D, et al. (2016) Educational Needs of Patients with Inflammatory Bowel Disease (IBD) and Non-Adherence to Medical Therapy-A Qualitative Study. J Inflam Bowel Dis \& Disord 1: 106.

Page 4 of 8

\begin{tabular}{|l|l|}
\hline Critical Understanding Area & Frequency of occurrence \\
\hline The importance of adherence to medication & 13 \\
\hline IBD in general* & 13 \\
\hline The patient's individual follow up plan & 9 \\
\hline Provider contact information & 2 \\
\hline Diet and nutrition & 1 \\
\hline The necessity of taking personal responsibility for one's own disease management & 1 \\
\hline The provider is the patient's advocate & 1 \\
\hline
\end{tabular}

Table 2: Patient identified educational needs and provider identified critical understanding areas for IBD patients.

"If you take the medications and the medications prove to be efficacious, their (the patient's) quality of life long term is just as if they didn't have the disease." (A provider explaining how important adherence to medication is for patient quality of life)

"It's the basic natural progression of this disease as far as how bad it could be or it could get." (A provider discussing the importance of patient understanding regarding IBD in general and how it can progress).

Providers also identified the follow-up plan as a critical area for patient education $(n=9)$.

"That the plan is in place that is agreeable to them and agreeable to the physician. That they understand what that plan is and where they are going."

Other respondents felt that ensuring patients know the provider's contact information is essential $(n=2)$. Furthermore, diet and nutrition $(n=1)$; the necessity of taking personal responsibility for one's own disease management $(n=1)$; and understanding that the provider is the patient's advocate $(n=1)$, were all conveyed as crucial patient comprehension areas.

"It is important for them (patients) to know that I am their advocate, and that they should share with me the struggles that they have, whether it is disease related or struggles with the side effects of medications, or even financial concerns about affording therapy."

\section{Barriers to desired information}

IBD patients and their providers identified numerous barriers to obtaining or conveying information that fulfilled patient educational needs (Table 3). Patients most frequently identified the variable quality of online information as a barrier $(n=12)$; whereas providers most commonly identified information oversaturation $(n=18)$.

"I don't trust anything that is on the internet." (A patient speaking in reference to the variable quality of available online information).

"Telling them [the patient] you have inflammatory bowel disease, there are two different kinds, here are all of the treatments, here are the risk factors, and adverse effects related to the treatments....that is information in a deluge for them (the patients)." (a provider explaining how information saturation is a barrier).
Additional barriers identified by patients included information oversaturation $(n=9)$, ineffective provider communication skills $(n=6)$, and lack of provider time to fully answer patient questions $(n=5)$.

"But you can't really take anything in...you shut down." (A patient discussing how it is difficult to process too much information).

"I had to go on WebMD and figure out what this disease is that this lady(the provider) is telling me I have." (A patient expressing frustration with the provider's communication skills).

"They have little interest in me-they literally give you 15 minutes of an appointment and outside of that they don't want to talk to you. Then they move on to the next person." (A patient explaining how lack of provider time is a barrier).

Other barriers conveyed by patients include: the inability to personalize the information $(n=2)$; information complexity $(n=2)$; erroneous information $(n=1)$; a lack of consolidated information $(n=1)$ and logistical constraints, such as inability to physically find the information $(\mathrm{n}=1)$.

"It (the available information) is generic; it's not exactly for you." (A patient describing the barrier of inability to personalize the information).

"Not being a trained professional, it is hard to decipher the information." (A patient explaining how information complexity is a barrier).

Providers also acknowledged that their lack of time was a significant barrier to information for patients $(n=11)$. Barriers specifically related to the healthcare system, such as difficulties with patient access to care, were also noted $(n=11)$.

"I think the most limiting factor is time." (A provider expressing lack of their time as a barrier for their patients).

"The difficulty is having patients be able to get into the system and talk about their issues." (A provider discussing the difficulties some patients experience accessing care)

Lack of consolidated information $(n=7)$; logistical constraints $(n=6)$; lack of provider support staff $(n=6)$; information complexity $(n=5)$; inability to personalize information $(n=2)$; ineffective provider communication skills $(\mathrm{n}=1)$; erroneous information $(\mathrm{n}=1)$; and language $(n=1)$, were other barriers to information that providers conveyed. 
Citation: Wheat CL, Maass M, Devine B, Thornton TY, Grembowski D, et al. (2016) Educational Needs of Patients with Inflammatory Bowel Disease (IBD) and Non-Adherence to Medical Therapy-A Qualitative Study. J Inflam Bowel Dis \& Disord 1: 106.

Page 5 of 8

"You can usually get the information somewhere, but the problem is, to be able to find the perfect resource in one place. It's very challenging." (a provider discussing how the lack of consolidated information is a barrier for both patients and providers).

"We don't have someone specifically that supports IBD patients here, unlike some other IBD clinics. They [specialized support staff] are very up on their IBD because that is all that they do." (A provider explaining how a lack of specialized support staff is a barrier for their patients).

"Sometimes the information, because it is FDA mandated, puts fear in patients that is not necessary." (A provider conveying how information complexity can be a barrier).

\begin{tabular}{|l|l|l|}
\hline Information Barrier & $\begin{array}{l}\text { Patient } \\
\text { identified }\end{array}$ & $\begin{array}{l}\text { Provider } \\
\text { identified }\end{array}$ \\
\hline The variable quality of online information available & 12 & 7 \\
\hline Information oversaturation & 9 & 18 \\
\hline Ineffective provider communication skills & 6 & 1 \\
\hline Lack of provider time & 5 & 11 \\
\hline The inability to personalize the information & 2 & 2 \\
\hline Information complexity & 2 & 5 \\
\hline Erroneous information & 1 & 1 \\
\hline Lack of consolidated information & 1 & 7 \\
\hline Logistical constraints & 1 & 6 \\
\hline Barriers that exist within the healthcare system & 1 \\
\hline Lack of support staff & 0 & 11 \\
\hline Language barriers & 0 & 6 \\
\hline
\end{tabular}

Table 3: Patient and provider identified barriers to desired information sources.

\section{Factors Associated with Patient Comprehension, Decision Making, and Non-Adherence}

Table 4 summarizes the identified factors that may impact patient comprehension and patient decision making.

\begin{tabular}{|l|l|l|}
\hline Patient comprehension & $\begin{array}{l}\text { Patient } \\
\text { identified }\end{array}$ & $\begin{array}{l}\text { Provider } \\
\text { identified }\end{array}$ \\
\hline Patient education level & 0 & 13 \\
\hline Social and cultural factors & 0 & 13 \\
\hline Language fluency & 0 & 11 \\
\hline Patient age & 1 & 8 \\
\hline Patient interest & 1 & 8 \\
\hline Patient health literacy & 0 & 7 \\
\hline Patient absorption time & 1 & 5 \\
\hline Patient disease severity & 1 & 3 \\
\hline Patient anxiety and/or fear & 0 & 2 \\
\hline Patient learning or mental disabilities & 0 & 2 \\
\hline Patient mental health & 0 & 1 \\
\hline Patient decision making & Patient & $\begin{array}{l}\text { Provider } \\
\text { identified }\end{array}$ \\
\hline
\end{tabular}

\begin{tabular}{|l|l|l|}
\hline $\begin{array}{l}\text { Short term versus long term effects of therapy } \\
\text { and/or disease }\end{array}$ & 4 & 5 \\
\hline Provider trust & 3 & 4 \\
\hline Insurance coverage and/or cost of treatment & 0 & 4 \\
\hline Patient past experiences & 7 & 2 \\
\hline Feeling as if there are no alternative choices & 2 & 1 \\
\hline Patient educational level & 0 & 1 \\
\hline Patient socioeconomic factors & 0 & 1 \\
\hline
\end{tabular}

Table 4: Factors that impact patient comprehension and decision making

\section{Comprehension}

Providers frequently indicated that patient educational level $(n=13)$, as well as social and cultural factors $(n=13)$, are associated with patient comprehension. In addition, language fluency plays a substantial role in patient understanding $(\mathrm{n}=11)$.

"the literacy level of some patients, even information from the CCFA (Crohn's and Colitis Foundation of America), is too high of a literacy level for them to understand." (A provider explaining how education impacts patient comprehension). 
Citation: Wheat CL, Maass M, Devine B, Thornton TY, Grembowski D, et al. (2016) Educational Needs of Patients with Inflammatory Bowel Disease (IBD) and Non-Adherence to Medical Therapy-A Qualitative Study. J Inflam Bowel Dis \& Disord 1: 106.

Page 6 of 8

"We do have a lot of people, who need translators, where English is not their first language, I think that it is even harder for them (The patients). To process all of the information." (A provider discussing how language influences patient understanding).

Other patient factors conveyed by providers as influencing patient comprehension included: age $(n=8)$; level of interest $(n=8)$; health literacy $(n=7)$; absorption time $(n=5)$; disease severity $(n=3)$; anxiety and/or fear $(\mathrm{n}=2)$; learning or mental disabilities $(\mathrm{n}=2)$; and mental health $(\mathrm{n}=1)$.

"Some people are less interested in the details of what is going on, and then some people are a lot more interested, and want to know a lot more about the details of their care." (a provider expressing how patient interest impacts understanding).

Patients identified fewer factors associated with information comprehension than providers. These factors included: age $(n=1)$; interest $(\mathrm{n}=1)$; absorption time $(\mathrm{n}=1)$; and disease severity $(\mathrm{n}=1)$.

"I was really, really ill, so was relatively incoherent, and was not in the ideal state of mind to be given that sort of information." (A patient explaining how disease severity plays a role in the comprehension of information).

\section{Decision making}

Providers identified a number of potential factors that may influence patient decision making. Balancing the short term versus the long term effects of therapy and/or the disease was the most frequently noted concern $(\mathrm{n}=5)$.

"I think that there are some misconceptions about the side effects of treatment, but also having long term, uncontrolled disease, even if they (the patient) don't have symptoms."

Trust in the patient's provider $(n=4)$; patient insurance coverage and/or the cost of treatment $(n=4)$; and patient past experiences $(n=2)$ were also commonly discussed.

"They (the patients) have a tough time trusting that we (the providers) are going to give them good care, and that they are going to be safe." (A provider discussing how it is especially challenging for patients who are transitioning from paediatric care to adult care, and how trust can influence the patient's decision making).
"Usually it is based on what the insurance has determined that the patient is going to get." (A provider explaining how the patient's insurance often dictates the patient's treatment course).

A few providers explained how educational level $(n=1)$; socioeconomic factors $(\mathrm{n}=1)$; and patients feeling that they lack alternative treatment choices $(n=1)$ all influence the patient's decision making process.

"I think that some of the fear is that there is nothing out there that is going to help" (a provider discussing how a patient believing that lacking alternative treatment choices can influence decision making)

Patients frequently discussed how their past experiences $(n=7)$ and balancing the short term versus long term effects from therapy and/or disease $(n=4)$ influenced their decision making.

"I have the risk of getting cancer, I have the risk of death, and it brings me to ask, which one is worse, really?" (a patient conveying how taking into account the shorter term potential effects from unmanaged disease versus the longer term potential risks from therapy impacts decision making).

Other patients expressed that trust in their provider $(n=3)$ and feeling as if there are no alternative choices $(n=2)$ significantly impact their decision making.

"I have no choice." (a patient expressing frustration at perceived lack of choice regarding receiving medication for their IBD)

\section{Patient non-adherence}

Table 5 describes the provider identified factors associated with patient non-adherence. Providers' most frequently expressed issue related to patient non-adherence was a patient's lack of current symptoms or denial of their chronic condition $(n=12)$.

"If you are not feeling well, I think it is in the forefront of your mind, but if those patients are in remission and are doing well, I think that they are more likely to not go to an infusion or miss a day of medication."

\begin{tabular}{|l|l|}
\hline Factor & Frequency of occurrence \\
\hline The patient feels well or is in denial of their chronic condition & 12 \\
\hline Social or cultural factors & 10 \\
\hline The patient has experienced side effects or is fearful of side effects & 9 \\
\hline Logistical factors & 9 \\
\hline The treatment course is complicated & 8 \\
\hline Lack of insurance, cost, or the patient has experienced an insurance change & 7 \\
\hline The patient has a lack of understanding regarding their disease & 7 \\
\hline The patient has a lack of understanding in general & 2 \\
\hline The patient is forgetful & 2 \\
\hline The patient has mental health issues & 1 \\
\hline
\end{tabular}


Citation: Wheat CL, Maass M, Devine B, Thornton TY, Grembowski D, et al. (2016) Educational Needs of Patients with Inflammatory Bowel Disease (IBD) and Non-Adherence to Medical Therapy-A Qualitative Study. J Inflam Bowel Dis \& Disord 1: 106.

Page 7 of 8

\begin{tabular}{|l|l|}
\hline The patient is embarrassed by their disease and/or its treatment & 1 \\
\hline The patient desires natural therapy & 1 \\
\hline The patient is fearful or has anxiety & 1 \\
\hline Lost or stolen medication & 1 \\
\hline
\end{tabular}

Table 5: Provider identified factors associated with patient non-adherence.

Social or cultural factors $(n=10)$; past side effects or fear of side effects $(n=9)$; logistical factors $(n=9)$; complicated treatment courses $(\mathrm{n}=8)$; financial considerations $(\mathrm{n}=7)$; and lack of patient understanding regarding IBD $(n=7)$ were other commonly noted themes regarding patient non-adherence.

"There are so many other things that they (the patients) have to worry about in their life that it is hard to remember when to take their pills." (A provider discussing how social factors influence patient adherence).

"Patients think oh well, I can't afford it so I just won't do it, so they stop and hope for the best." (a provider conveying how insurance and cost impact patient adherence).

"It is difficult to bridge that gap of, even if you can't see the damage that is being done it is really important for you to maintain your medication course." (a provider explaining how it is difficult for some patients to truly understand their disease and its effects).

Some providers felt that patient lack of understanding in general $(n=2)$; forgetfulness $(n=2)$; mental health issues $(n=1)$; embarrassment by their disease and/or its treatment $(n=1)$; desire for natural therapy $(\mathrm{n}=1)$; fear and/or anxiety $(\mathrm{n}=1)$; and lost or stolen medication $(\mathrm{n}=1)$ were other factors that influence patient adherence.

"They (the patients) are scared because these are scary medications."

\section{Discussion}

The perspectives of the patients and providers interviewed for this study demonstrate several key issues related to IBD patients' educational needs and barriers to obtaining desired information. We also identified factors perceived to be involved with patient comprehension, decision making, and non-adherence to provider treatment recommendations. First, despite the current availability of many educational resources for IBD patients, there remain a number of unmet educational needs for this population. Patients want to know how to prevent the symptoms of their disease, receive information regarding the cause of IBD, and have more discussion around the disease in general. A number of patients also expressed an interest in diet and nutrition, specifically how to maximize nutritional status given their disease. These patient concerns overlapped with provideridentified critical understanding areas, particularly concerning diet and nutrition and information regarding IBD in general. As such, these areas may be targets for novel educational resources to improve patient understanding. Exploring existing barriers to patient understanding may also assist in developing needed educational resources. Many patients utilize the internet as a primary information source, but the variable quality of online information available is a significant barrier. Patients and providers both identified information oversaturation, ineffective provider communication skills, and lack of provider time as additional information barriers for IBD patients. These results highlight the need for providers to consider both the amount and format of information presented to patients at any given time, particularly for newly diagnosed patients and for those who are severely ill. Furthermore, mechanisms to increase the provider time available to IBD patients, such as the availability of specialized support staff, are crucial to patient education and understanding. Our findings are similar to those in the published literature. Information on the prevention of symptoms, complications, causes, long term prognosis and/or outcomes, effects and/or risks from therapy, and alternative treatments were identified as educational needs both by our subjects and in prior studies $[8-13,17,18]$. Education regarding diet and nutrition was identified by many of our patients as an area where they desired additional information; however this was not a previously identified concern in this population. This dissimilarity may be specific to our population or an emerging area of educational need that has yet to be fully explored. Our study is unique in its evaluation of both patient educational needs and areas where providers feel that patient understanding is critical. Factors that are associated with patient comprehension and decision making are essential considerations when discussing strategies to meet the educational needs of IBD patients. Providers recognized patient educational level, social and cultural factors, language, patient age, level of interest, health literacy, and absorption time as determinants of patient comprehension. Patients agreed that their age, interest, absorption time, and disease severity all play a role in understanding. Balancing the short term versus the long term effects of therapy and/or IBD, trust in the patient's provider, and the patient's past experiences were commonly identified by both patients and providers as factors that significantly influence patient decision making. Therefore, it is critical to ensure that educational resources are appropriate for varying health literacy levels and age groups. In addition, resources must be available in multiple languages and be developed by individuals with cultural awareness of the groups being targeted. Identifying issues associated with patient nonadherence that are amenable to change is also important for targeting educational interventions. Two recent reviews emphasize the importance of considering IBD patients' beliefs and concerns to effectively evaluate and address physical and perceptual barriers to adherence $[19,20]$. In addition, more research is needed to determine the predictors of nonadherence so that providers can intervene $[19,20]$. We found the issues that providers most commonly identified as associated with patient non-adherence could be modified through education targeted towards IBD, its effects, and its management. Patient self-management programs are effective interventions for individuals with other chronic diseases such as diabetes, and could be used as models for use in the IBD population. These programs are typically led by trained lay leaders or health care professionals. They have a group based format and content areas include: symptom management; understanding medication use; solving health-related problems; and communication with providers [21-23] Prior nurse-led patient-support programs that target an individual patient have not 
Citation: Wheat CL, Maass M, Devine B, Thornton TY, Grembowski D, et al. (2016) Educational Needs of Patients with Inflammatory Bowel Disease (IBD) and Non-Adherence to Medical Therapy-A Qualitative Study. J Inflam Bowel Dis \& Disord 1: 106.

Page 8 of 8

been shown to improve adherence in IBD patients [24]; however, selfmanagement programs have a different structure. There is minimal research into the effectiveness of self-management interventions in IBD; however the available evidence suggests that self-management programs are associated with a number of positive outcomes [22]. Selfmanagement programs tailored towards IBD patients are able to not only meet many of the patient educational needs identified in our study, but are able to circumnavigate the barriers to information and patient adherence identified here. These programs can also take into account the varied factors associated with patient comprehension and decision making. Thus, they are potentially effective educational interventions that warrant further study. This study has several important limitations. First, we interviewed a small number of patients and providers in one urban area. Although we reached information saturation with our sample, the overall number of participants was low, and this may limit the generalizability of our findings. In addition, patient and provider interviewees were recruited from academic affiliated practices; thus our findings may be dissimilar to the perspectives of patients and providers in non-academic affiliated practices. Furthermore, many of our patients were exposed to biologic medications which are typically prescribed for moderately to severely active disease; consequently our results may not be representative of patients with milder disease. Further study of large and diverse populations will improve the generalizability of the findings. Poorly controlled disease in IBD patients increases the risk for serious negative outcomes, and a significant contributing factor to suboptimal disease management is patient non-adherence to medical therapy. Our findings highlight existing knowledge deficits among the IBD patient population, and the barriers to IBD patient comprehension and decision making. In addition, we identified many potential influences to patient non-adherence that might be amenable to change through education. Exploration of the areas associated with patient nonadherence that are mutable will assist in developing targeted educational resources. Tailoring these resources to patients at higher risk for non-adherence may maximize their impact upon patient understanding, decision making, and outcomes.

\section{Acknowledgment}

We wish to express our gratitude to all of patients and providers who participated in this study at Harborview Medical Center, University of Washington Medical Center, and the VA Puget Sound Health Care System.

\section{References}

1. Loftus EV, Crowson CS, Sandborn WJ, Tremaine WJ, O'Fallon WM, et al. (2002) Long-term Fracture Risk in Patients with Crohn's Disease: A Population Based Study in Olmsted County, Minnesota. Gastro 123: 468-475.

2. Peyrin-Biroulet L, Loftus EV, Colombel JF, Sandborn WJ (2011) Long Term Complications, Extraintestinal Manifestations, and Mortality in Adult Crohn's Disease in Population Based Cohorts. Inflamm Bowel Dis 17: 471-478.

3. Bernstein CN, Blanchard JF, Houston DS, Wajda A (2001) The Incidence of Deep Venous Thrombosis and Pulmonary Embolism Among Patients with Inflammatory Bowel Disease: A Population Based Cohort Study. Thromb Haemost 85: 430-434.

4. Bernstein CN, Wajda A, Blanchard JF (2008) The Incidence of Arterial Thromboembolic Diseases In Inflammatory Bowel Disease: A Population Based Study. Clinical Gastroenterology and Hepatology 6: 41-45.
5. Farraye FA, Odze RD, Eaden J, Itzkowitz SH (2010) AGA Technical Review on the Diagnosis and Management of Colorectal Neoplasia in Inflammatory Bowel Disease. Gastro 138:746-774.

6. Kane SV, Cohen RD, Aikens JE, Hanauer SB (2001) Prevalence of Nonadherence With Maintenance Mesalamine in Quiescent Ulcerative Colitis. Gastroenterology 96: 2929-2933.

7. Greenley R, Kunz J, Walter J, Hommel K (2013) Practical Strategies for Enhancing Adherence to Treatment Regimen in Inflammatory Bowel Disease. Inflamm Bowel Dis 19: 1534-1545.

8. Bernstein K, Promislow S, Carr R, Rawsthorne P, Walker J, et al. (2011) Information Needs and Preferences of Recently Diagnosed Patients with Inflammatory Bowel Disease. Inflamm Bowel Dis 17: 590-598.

9. Becker H, Grigat D, Ghosh S (2015) Living with inflammatory bowel disease: A Crohn's and Colitis Canada Survey. Can J Gastroenterol Hepato 1 29: 77-84.

10. Catalan-Serra I, Huguet-Malaves J, Minguez M, Torrella E, Paredes JM, et al. (2015) Information resources used by patients with inflammatory bowel disease: Satisfaction, expectations, and information gaps. Gastroenterol Hepatolo 38: 355-363.

11. Pittet V, Vaucher C, Maillard M, Marc Girardin, Gerhard Rogler, et al. (2015) Information needs and concerns of patients with Inflammatory Bowel Disease: What can we learn from participants of a national clinical cohort? J Crohns Colitis.

12. Baars J, Markus T, Kuipers E, van der Woude CJ (2010) Patients'Preferences regarding Shared Decision-Making in the Treatment of Inflammatory Bowel Disease: Results from a Patient-Empowerment Study. Digestion 81: 113-119.

13. Conrad S, Huppe A, Raspe H (2012) Preference of Patients with Inflammatory Bowel Disease Regarding Information and Shared Decision-Making: Results from a Cross-Sectional Survey in Germany. Z Gastroenterol 50: 364-372.

14. Guest G, Bunce A, Johnson L (2006) How Many Interviews Are Enough? An Experiment with Data Saturation. Field Methods February 18: 59-82.

15. Bernard H, Ryan G (2010) Analyzing Qualitative Data: Systematic Approaches. Thousand Oaks, CA: SAGE Publications.

16. Pope C, Ziebland S, Mays N, Analysing Qualitative Data. BMJ 320: 114-6.

17. de Rooy E, Toner B, Maunder R, Greenberg GR, Baron D, et al. (2001) Concerns of Patients with Inflammatory Bowel Disease: Results From a Clinical Population. Am J Gastroenterol 96: 1816-1821.

18. Keeton R, Mikochka-Walus A, Andrews J (2015) Concerns and worries in people living with inflammatory bowel disease (IBD); A mixed methods study. J Psychosomatic Research 78: 573-578.

19. Herman ML, Kane SV (2015) Treatment Nonadherence in Inflammatory Bowe Disease: Identification, Scope, and Management Strategies. Inflamm Bowel Dis 21: 2979-2984.

20. Kane SV, Robinson A (2010) Review article: understanding adherence to medication in ulcerative colitis - innovative thinking and evolving concepts. Aliment Pharmacol Ther 32: 1051-1058.

21. Bodenheimer T, Lorig K, Holman H, Grumbach K (2002) Patient Selfmanagement of Chronic Disease in Primary Care. JAMA; 288: 2469-2475.

22. Barlow C, Cooke D, Mulligan K, Beck E, Newman S, et al. (2010) A Critical Review of Self-Management and Educational Interventions in Inflammatory Bowel Disease. Gastroenterol Nurs 33: 11-18.

23. Ory M, Ahn S, Jiang L, Smith ML, Whitelaw N, et al. (2013) Successes of a national study of the Chronic Disease Self-Management Program: meeting the triple aim of healthcare reform. Med Care 51: 992-998.

24. Moss AC, Chaudhary N, Tukey M (2010) Impact of a patient-support program on mesalamine adherence in patients with ulcerative colitis -- A prospective study. J Crohns Colitis 4: 171-175. 